\title{
EFFECTS OF WORKFORCE DIVERSITY ON TEAM PERFORMANCE AT MOI TEACHING AND REFERRAL HOSPITAL
}

\author{
Anne Kalei \\ Lecturer, Machakos University, SoB, Kenya \\ Demian Matelong \\ Lecturer, JKUAT, SoB, Eldoret, Kenya
}

\begin{abstract}
Dealing with increasing level of workforce diversity that is within modern employee management of diversity has been progressively a matter of interest to many organization's management programs that are adopted steadily. The purpose of the study was to establish the effects of cultural diversity on team performance. The study was guided by social identity theory and symbolic interactionist theory. The study adopted a descriptive survey research design. The research targeted 373 respondents comprising of top managers, middle level managers and other employees from Moi teaching and referral hospital Eldoret. For the purpose of getting a representative sample, the researcher used a purposive sampling method to sample top and middle level managers and simple random technique to sample other employees. Questionnaires were used as data collection instruments. A Cronbach's alpha was used to assess the reliability coefficient of the research instruments. The instruments were pre-tested to improve its validity and relevance to the objectives of the study. The questionnaires were scrutinized for errors and omissions, ambiguity, legibility and relevance. The data was analysed using regression model and presented in tables and charts. The findings of this study indicated that the study variable cultural diversity $(p=0.000)$ had a significant relationship with team performance. Based on the finding of this study, it is recommended that Moi teaching and referral hospital Eldoret to achieve a high level of efficiency and effective in service provision, the management in the company must ensure that adoption of diverse workforce strategies. The study suggests that further research be done on the same field but in other sectors or organizations also further research should focus on other aspects of workforce diversity.
\end{abstract}

Key Words - workforce diversity, team performance, age diversity, cultural diversity, ethnic diversity

Background of the Study

Dealing with increasing level of workforce diversity that is within modern employee management of diversity has been progressively a matter of interest to many organization's management programs that are adopted steadily. Barker, Hartel (2004), suggested that diversity is the power or force to realize the potential of workers or employees of any organization. It is very vital in ensuring that the organization gains its objectives and results. Improvements in systems and globalization has led the world to be a global village hence, fostering interactions of different people from different cultural backgrounds, ethnic, education and age to form a diverse workforce. In this regard, organization systems and entities are researching on ways in which to better serve the diverse group. This includes being able to attract and retain the best and most qualified workers (Barker, Hartel 2004)

Globally, managing diversity is so much crucial because it is a factor which enables the organization to have a better competitive advantage and yield positive results. For instances in Pakistan, a lot of changes have been evident because of the nature of their labour market and other economic conditions. Many females after advancing in their university education don't stay at home as it used to be but rather go beyond and look for job opportunities in order improve their economic standards to from their families at large. This is the reason as to why there has been a positive increase of women participating in employment opportunities. The percentage increased in the year $2010-2011$ by $22.2 \%$. Pakistan still face a challenge of workforce diversity in that still as per now there are still some other jobs that are only left for men alone as compared to developed countries where jobs opportunities are open to either gender 
In Nigeria the issue of workforce diversity has not been well understood and given attention by companies, organizations or institutions due to a culture that has dominated the country which is demonstrated by socio-political patronage of workforce in leadership mostly in the human resource, procurement and administration. However other institutions and corporate organizations with broad ideas about their market niche have started understanding hence giving attention to the same. Research has been conducted in recent years over workforce diversity and its effects on organizational but very few have actually demonstrated and examined the impacts that it can bring to the organization (Herring, 2005).

Due to globalization many organizations in developed and developing countries have embraced diversity amongst their workers which is most challenging issue in the human resource sector. Researchers have gone further to fully understand impact of diversity and its management on organizational output. Research stipulates that effects of workforce diversity have got contradictory duplex results on organizational success. Further studies also illustrate that several classifications and forms of diversity are attributed to high performance, innovation and high level of decision making. Other research demonstrates that greater diversity increase chances of conflict, minimizes social adaptability and raise the rate of employee turnover within the organization (DeVeale \& Manea, 2007)

Contemporary workforce in organization comprises of different people from different background and different cultures due to increase mobility, immigration and enhancement of technology. When dealing with increasing level of diversity inherent within the modern personnel, diversity management programs that are adopted has been steadily growing which leads to a diverse workforce (Hultsman, 2005). The current trends in the human resource; ageing workforce, the growing number of women joining the job market and the upcoming young generation in developed and developing countries has rendered institutions and organizations to change their way of life to suit the current workforce unlike the past

However, in Kenya, the quality assessment and performance improvement strategy report (2014) demonstrated that The Kenya School of Government had issues in regard to its employees. The employees had started to illustrate low morale which translated to low productivity hence leading to a reduction of customer satisfaction by $73 \%$ in the year 2012/2013 to $65 \%$ in the year 2013/2014.Contrastingly the report gave some recommendations that organizations must change its human resource strategy but more precisely on training of its workers on current issues, do away with impartiality nepotism and unfairness on their workplaces Barker, Hartel (2004) stated that embracing diversity in any organization is a key tool in sustaining the organization to its full ability. It will enable the organization to fully utilize the untapped talents gifts and ideas that are possessed by employees which will enhance the organization or business to expand and venture into new markets (Mukherjee, 2005).

In these regard the study helps to identify the effect of workforce diversity toward team performance in Moi Teaching and Referral Hospital. (Hornby 2005) defines workforce diversity as the total similarities and differences of people working in a particular institution or organization. A diverse workforce for instance, includes gender, age, ethnicity, and education background. Roberson, Kulik, \& Pepper (2003) illustrates that diversity in the workplace has positive results in terms of performance and policies.

\section{Statement of the Problem}

Lack of unity, cooperation, conflicts and negative attitude among workers has become a concern in many organizations. This has led to low morale of workers which at the end translate to low performance with negative performance index. Cooperate profitability or team profitability is dampened because of not embracing diversity. Organizations and institutions that have an objective of achieving its mission and goals must ensure that diversity management is part and parcel of its day to day agenda (Ely, \& Thomas, 2001) According to Lauby, (2005) Diversity management and workforce diversity is an integration that can create havoc and conflict if management is not geared towards the discipline of diversity management and its rationale. Therefore, most managers do not know on how to implement policies and regulations on diversity management in the workplace. As much as companies are training employees on issues of embracing differences, anti-harassments training, ethics, employees still do not adhere to those rules hence breaking them with their behaviour when it comes to diversity (Wood, \& Sangster, 2002) Research in recent years by (Larson, 2013) highlighted on gaps that existed between doctors and nurses. A systematic hierarchy always depicts that doctors are in the top then followed by nurses. In most cases there have been conflicts and communication failures amongst the two groups with medical errors which have adversely affected the performance. Employee diversity can damage morale, communication and can possibly increase turn over in an organization if unmanaged properly. Moi Teaching and referral hospital; have a workforce that is heterogeneous comprising of different tribes, culture, gender mix, age, religious beliefs and different conceptions. Therefore, the management of 
MTRH has a big task in ensuring that diversity is embraced in order to insulate the hospital from unmanaged diversity which can bring harm and hence possibly do the opposite of its mission and objectives. This can translate to conflicts reducing teamwork among the employees. Dahm (2003) did a study on the effects of workforce diversity towards the employee performance in an organization and found out that a comparison of performance of the individual employee had no comparison based on team performance. Moi teaching and referral hospital has been experiencing some problems which has led to poor performance in recent years (Webber, 2013). However, the cause might be attributed to other factors but this study is geared towards studying the effects of workforce diversity on team performance at MTRH with an in-depth of looking into elements of diversity such as cultural diversity, age diversity and ethnic diversity.

\section{Objective of the Study}

The objective of the study was to find out the effects of cultural diversity on team performance at Moi teaching and Referral Hospital

Significance of the Study

The study will be important not only to Moi teaching and referral hospital but also other processing companies in this sector and to larger extent managers of other industries. It will help them understand the effects of diversity on team performance in order to strategically plan on the practices to employ in their internal human resource management systems. The study will also highlight other important relationships that require further research; this will be in the areas of management of human resource.

The findings of this study will help the management of Moi Teaching and Referral Hospital to fully understand appreciates and manages workforce diversity and strengthens the collaboration and relationship between doctors and nurses to improve efficiency in the hospital.

Finally, the study will contribute to the academic literature and knowledge to other researcher who wish to expand on the issue of workforce diversity other variables, it would add to the existing literature, as it will be point of reference for students

Scope of the study

The study was carried out in Moi Teaching and Referral Hospital, Uasin Gishu County. The study period was between June 2021 and August, 2021. Moi Teaching and Referral Hospital (MTRH) is the second National Referral Hospital in Kenya. It is located in Eldoret, in the Rift Valley Province of Kenya. It was opened in 1917 as a cottage hospital and it was not until the establishment of Moi University in 1984 and the subsequent establishment of the Faculty of Health Sciences at the University that the hospital elevated from a district hospital to a teaching and referral institution The study was conducted in the human resource departments in Moi teaching and referral hospital Eldoret. The study focused on the effects of cultural diversity on team performance in Moi teaching and referral Hospital Eldoret. The respondents of this study were managers, departmental heads and other employees.

\section{Literature Review}

The study informed by the following theories:

Symbolic Interactionist theory

Symbolic Interactionist theory is an individual centred orientation theory that focuses on individuals in interaction and within a group, and on the composition development of individual personality. Its theory is employed in this study because of the focus on the employee experience of diversity resulting from interactions in the workplace. This perspective has its roots in the pragmatist philosophies of Mead, Dewey, Thomas and Park of the so-called Chicago School (Reskin, 2003).

The method advocated by symbolic interactionism is to look at the process through which individuals define the world from the inside and at the same time identify their world of objects. This predominantly small scale theory focuses on interactions and individual personalities. It is a subject-orientated approach that emphasizes the subjective intentions and orientations of individuals in relation to their wishes, motivations, wants and situations. In addition to their interpretation and understanding of symbolic interactionism is the reflexive nature of human action. It assumes that human nature is not motivated solely by external and internal factors, but rather through meaningful, reflexive interactions between individuals. The Strategic Diversity management approach is an integration of the social theory, management theory and psychology which all converge on both the positive and negative effects of workforce diversity. Graen rightly notes that diversity may be an asset or liability depending on how it is integrated into the organization. DeVeale and Manea (2007) stated that reliance on surface level diversity tends to produce dysfunctional results for both individuals and their organizations than deep level diversity. Understanding the preceding theories is helpful for the study, which will introduce research on factors affecting management of workforce diversity

Social Identity Theory

Social identity theory asserts that team membership creates group and improvement in ways in which itfavor the in-group at the expense of the out-group (Ferner, Almond \& Colling, 2005) showed that the mere act of people categorizing themselves as team 
members was enough to guide them to show team favouritism. When being categorized in a team membership, people look at achieving positive selfesteem by completely differentiating themselves in groups to form a comparison in some valued dimension. This theory has emphasized more on the meanings related to being a member of a social category; it is cantered a lot on the meanings related to doing a job. Social identity theorists have argued that as a result of individuals outlining themselves in terms of their social group membership and enact roles as part of their acceptance of the normative expectations of in group members, the conception of role is subsumed underneath the conception of team (Ferner, Almond \& Colling 2005). However, social identity theorists have usually not cantered on these roles. Given their bigger emphasis on team identification, they need to focus a lot on cognitive outcomes like ethnocentrism team polarization and team cohesiveness (Reskin, 2003). Identity theorists have centered more on the behaviours created by persons as a function of their role identities.

\section{Empirical Review}

The idea of diversity has been observed as a recent and novel tool and thus the literature in management of manpower diversity has been growing in recent years. In line with the study by (Ely, Thomas 2001), the results of various achievement policies on employee performance. The study showed that diversity will improve performance and recruitment from a wider varied and quality offers the firm a bigger talent pool. Ely, Thomas (2001), additionally it is seen that increasing diversity expands the breadth of views and ideas offered to organizations in creating selections which cultural diversity, academic, individual personalities and skilled background will influence the different depth of information use.

Various studies like factors affecting diversity management in organizations has been done by Kioko (2010) and showed that the majority respondents did not have a written policy or program that features the idea of worker diversity within their organization whereas solely some respondents in the study indicated that their organizations were discussing adding such a policy or program. Regarding the structure activities that were enclosed within the diversity program or policy for those firms that had different program, the mostly reported activities were achievement. This shows that there's still a long way to change into activities of workforce diversity programs and it is clear that discussing diversity alone in terms of gender and race discrimination will cause employee anger that may influence morale, turnover, and performance.

Effects of cultural diversity on team performance
Business organizations and educational establishments are sure-fire solely to the extent that they ready to embrace and encourage manpower diversity. By guaranteeing that their work is considerably various which minority teams are a unit revered, appreciated, and valued, the educational environments are ready to harness the collective information of their organization. As economic expert Crouse, (2005) notes, the collective information of organizations, as well as educational establishments, will be increased by as well as individuals with completely different experiences, trainings, and cultural backgrounds.

Cultural and national diversity in organizations will be seen from a negative view from social identity and similarity-attraction or an optimistic read competitive that cultural diversity facilitates processing of information, learning, and drawback determination capability (Cox et al., 1991; Ely \& Thomas, 2001). Whereas the logic for the positive and negative effects of cultural diversity is obvious, the definition, activity, and empirical examination of the results of cultural diversity in organizations area true challenge. According to Schuller (2009), some staff understands company life as insensitive to their culture. To counteract these feelings of alienation, high management at several companies has been fitting support teams. These teams are meant to provide a nurturing climate for staff support teams on its website.

\section{Sample Size}

To get a representative sample, the researcher used purposive sampling method to sample top managers and middle level managers of Moi teaching and referral hospital, the process whereby the researcher selects a sample based on experience or knowledge of the group to be sampled. To select other employees, the researcher used simple random sampling technique. Therefore, 9 respondents were sampled from top management, 32 from middle level management and 332 other employees making a total of 373 respondents. The researcher obtained permission from the Management of Moi teaching and referral hospital before data collection, and personally administered the questionnaires to the respondents leaving them individuals to fill then collect them later.

\section{Data Collection tool}

The study used questionnaire; this is a collection of items to which a respondent is expected to react in a written form and it is a primary source of collecting data. The designed questions or items in word format were distributed to the respondents. This method collects a lot of information over a short period of time. The method is suitable when the information 
needed can be easily described in writing and if time is limited. In this study, the respondents were given enough time to complete the copies of the questionnaire before returning them for analysis. The questionnaire included structured questions. This allowed the respondents to give their own views.

Reliability of Research Instruments

Reliability is concerned with precision and accuracy. For research to be reliable it must demonstrate that if it is to be carried out on a similar group of respondents in a similar context (however defined), then, similar results would be found. Poor reliability degrades the precision of a single measurement and reduces ability to track (Orodho, 2003). A pilot study was conducted in Uasin Gishu County hospital a month before the actual study. The constructs testing for reliability was achieved by calculating the Cronbach's alpha. Higher alpha coefficient values mean that scales are more reliable. Mugenda, (2003) says that any value above 0.7 is considered appropriate threshold.

\section{Validity of Research Instruments}

The research instrument was pre-tested to improve its validity and relevance to the objectives of the study. The questionnaires were scrutinized for errors and omissions, ambiguity, legibility and relevance. The questionnaires' content, structure and sequence was then appropriately amended to remove any ambiguities and to enhance content validity. To determine the content validity of the questionnaire items, research experts were used to examine them and their suggestions and comments were used as a basis to modify the research items.

Data Analysis and Presentation

The data for the study was entered and coded for completeness and accuracy of information with the help of SPSS version 22, data was analysed by tabulating the data using frequency tables and correlation. The data was analysed using both inferential and descriptive, Descriptive statistics included frequencies, percentages, tables and charts, while for inferential statistics; Multiple regression was used for comparisons of the variables. Multiple regression has the following assumptions, Normality assumption where multiple regression assumed that all the variables have normal distribution (Kothari, 2010). Kolmogorov-Smirnov test (K-S) one sample test was used to test normality of assumption. Linearity assumption; in this study it will be assumed that the relationship between variables is linear. Linearity was tested by use of Pearson correlation. Homoscedasticity means that the variance of errors is the same across all levels of the independent variables. This assumption can be checked by Durbin Watson test.

\section{RESEARCH FINDINGS AND DISCUSSIONS}

Among the 373 questionnaires distributed, 289 were duly filled and returned for analysis. This represented a response rate of $77.5 \%$. According to Mugenda and Mugenda (2003), a response rate of $50 \%$ or more is adequate. The authors asserted that return rate of $50 \%$ are acceptable to analyse and publish, $60 \%$ is good and $70 \%$ is very good. Based on these assertions from renowned research academicians, the responses rate for this study of $77.5 \%$ was considered to be adequate in forming conclusions and generalization of the study population.

\section{Gender of respondents}

The gender of the respondents in Figure 4.1 indicates that $56.1 \%$ constituted of male respondents while $43.9 \%$ comprised of female respondents. From this conclusion it was noted that majority of employees were male respondents. This implied that the study was not gender biased thus the variations in gender were not so significant to warrant the researcher to assume that the research responses were biased to a given gender. This also indicated that Moi Teaching and referral hospital meet the gender rule as stated by the Government of Kenya stating that not more than two-thirds of employees in an organization shall be of the same sex.

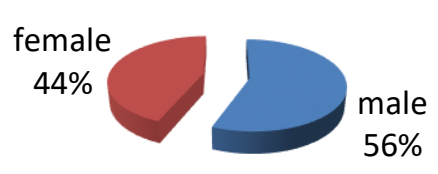

\section{Figure 4.1 Gender of Respondents}

Work experience

On the respondents work experience, the study indicated that Most of the staff had been in their current employment for between 6-10 years $32.2 \%$, those who had worked for between below 5 years with $23.5 \%$, then those who had worked for 11-15 years $30.8 \%, 16-20$ years was represented by $10.7 \%$ and lastly those who had worked for more than 21 years $2.8 \%$. This implies that majority of respondents 


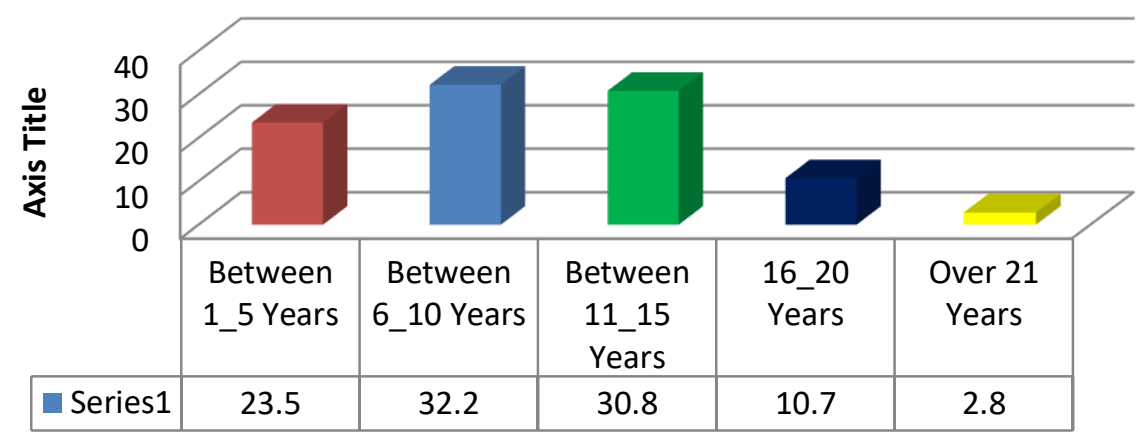

Figure 4.2 Work experience

\subsubsection{Highest Education Level}

Education level has an impact on how people respond to different opinions. The study sought to establish the education level of the respondent. The finding reveals that $4.5 \%$ of the respondents are a certificate holder that is KCPE and KCSE, $42.9 \%$ are diploma holders, $44.3 \%$ degree holders and $24 \%$ are master degree holders. This shows that majority of the respondent were degree holders, followed by diploma, this implies that the respondents are educated thus they could read, understand and interpret questionnaires reliably making data collected to be reliable.

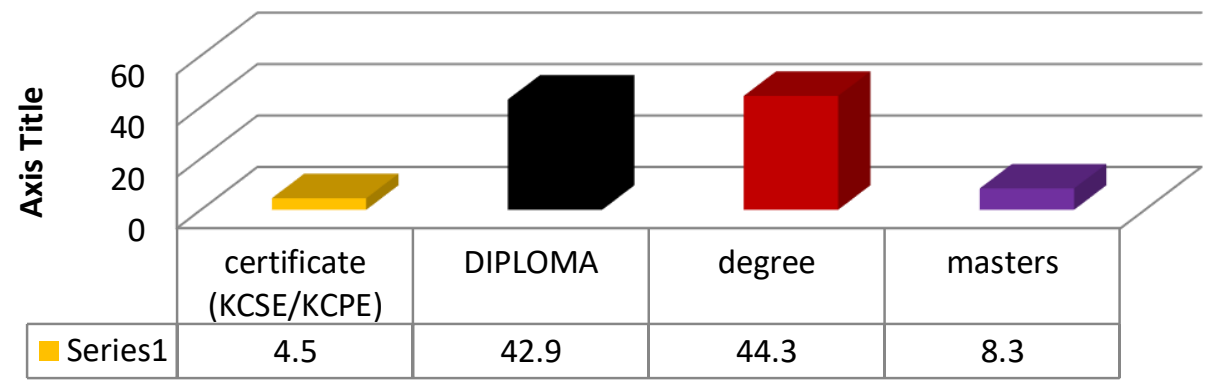

\section{Figure 4.3 Respondents on Highest Educational Level}

Descriptive statistics

The study sought to investigate the effects of workforce diversity on team performance: a case of Moi Teaching and Referral Hospital. The following tables give a breakdown of the descriptive measures of the items that was tested by the questionnaire. The analysis was done using descriptive statistics mean and standard deviation with a Likert scale of 1-5, The scores with a weak association have been taken to present a variable which had a mean score of 0 to 2.5 on the continuous Likert scale. On the other hand, scores of 2.5 to 3.5 represented moderate significance and mean scores of 3.5 to 4.8 on the continuous Likert was taken to be a great and strong effect, this is according to Parasuraman et al. (1998) who indicated that elements shows the lowest mean being 2 yet minimum score should be 3 and the maximum to be 5 . While for standard deviation of greater than 0.5 was evaluated to indicate homogeneity and a standard deviation less than 0.5 indicates heterogeneity of data.

Effects of cultural diversity on team performance The study sought to find out the effects of cultural diversity on team performance. This is shown in table 4.1 
Table 4.1 Effects of cultural diversity on team performance

\begin{tabular}{llc}
\hline cultural diversity and team performance & M & SD \\
\hline Minority groups are respected by the institution an positively influence diversity & 3.79 & 1.320 \\
& & \\
Company vision has an influence on diversity & 3.69 & 1.158 \\
& & \\
Company values influences diversity & 4.10 & .936 \\
& & \\
Company norms affects diversity & 4.04 & 1.051 \\
Company beliefs influences diversity & 3.53 & 1.539 \\
Habits of the company employees affects diversity & 3.97 & 1.194 \\
\hline
\end{tabular}

From the study findings, the respondents indicated that minority groups are respected by the institution an positively influence diversity, this was supported by a mean of 3.79 and a standard deviation of 1.320 , on whether the Moi teaching and referral hospital vision has an influence on diversity, the response was supported by a mean of 3.69 and a standard deviation of 1.158 , on the issue that company values influences diversity the mean was 4.10 spread at a standard deviation of 0.936 ,Further on whether the company norms affects diversity the mean was 4.04 and a standard deviation of 1.051 , on whether Company beliefs influences diversity the mean was 3.53 with a standard deviation of 1.539.Lastly when asked whether habits of the company employees affects diversity, the respondents agreed at mean of 3.97 spread at standard deviation of 1.194.. This implies that all the respondents were in agreement that cultural diversity is significant for organizational team performance since their responses were between $3.50-4.49$ implying a Strong relationship between the variables. Additionally, the standard deviation was between 0.9 to 1.2 which is higher than 0.5 indicating the homogeneity of study data.

These findings concur to that of Kunze, Boehm, \& Bruch (2009). Who indicated that managing workforce diversity effectively has a positive effect on competitive advantage. Competitive advantage is a strategy that gives an organization a distinctive competence. This competence and advantage stem from the process in which the management of diversity positively affects organizational behaviour and effectiveness. Further Kreitner and Kinichi, (2004) indicated that effectiveness in cultural diversity entails doing things right in an organizational performance factor management of workforce diversity is therefore paramount in improving performance and lowering costs.

This results are supported by social identity theory asserts that team membership creates group and improvement in ways in which it favours the ingroup at the expense of the out-group. This theory has emphasized more on the meanings related to being a member of a social category; it is centred a lot on the meanings related to doing a job. Social identity theorists have argued that as a result of individuals outlining themselves in terms of their social group membership and enact roles as part of their acceptance of the normative expectations of in group members, the conception of role is subsumed underneath the conception of team.

\section{Inferential Statistics}

Multiple regression model was used as a form of inferential statistics analysis to determine the relationship between the dependent and independent variables.

Regression analysis

The study also employed multivariate regression model to study the effects of workforce diversity on team performance. The dependent variable was the team performances and the independent variables were age diversity, cultural diversity and ethnic diversity.

\section{Conclusion}

The study concluded on the effects of cultural diversity on team performance that Minority groups are respected by the institution and positively influence diversity, Company vision, Company values, Company norms, Company beliefs and 
company habits affects diversity. To Miner, (2007), diversity can be classified into categories namely; primary and Secondary diversity. Primary diversity includes age, gender, sexual orientation and so on, which actual illustrates the main differences between various individuals. These primary differences also have the most impact on initial encounters and can be easily noticed and serve as filters through which people view.

\section{Major Recommendations of the Study \\ i. Moi teaching and referral hospital Eldoret to achieve a high level of efficiency and effective in service provision, the management in the company must ensure that adoption of diverse workforce strategies. \\ ii. That management of Moi teaching and referral hospital Eldoret should support workforce diversity efforts such as mentoring or recruitment of minorities for top jobs, organizational diversity and upward mobility efforts more effectively; this will promote organizational team performance. \\ iii. Further the management of Moi teaching and referral hospital Eldoret should ensure effective communication of the outcomes and future goals of the diversity strategy; they should also have expanded diversity programmes beyond employee groups to include additional stakeholders and the public. \\ iv. Lastly the organization should recruit its employees from diverse backgrounds, recruitment in workforce diversity will enable the hospital to select competent, qualified and knowledgeable workforce that improve on its productivity and improve the performance of the company.}

\section{REFERENCES}

Allison, M. (2009), Leisure and quality of life: Issues facing racial and ethnic minority elderly Therapeutic Recreation Journal. (pp. 59-60).

Anderson, J. A. (2012). Thinking about Diversity, Training and Development, Jossey-Bass Publishers, (pp. 49, 59-60).

Argyris, C. (2006), Knowledge for action: A guide to overcoming barriers to organizational change. San Francisco: Jossey-Bass Publishers, ISBN-1-55542519-4, (pp. 309).

DeVeale, C, and Manea, L. (2007), Companies embrace power of diversity. Canadian HR Reporter, (pp. 14-15).
Ely, R. J., and Thomas, D. A. (2001) Cultural diversity at work: The effects of diversity perspectives on work group processes and outcomes, (pp. 46, 273).

Ferner, A., Almond, P. \& Colling, (2005). Institutional theory and the cross-national transfer of employment policy: The case of 'workforce diversity' in US multinationals. Journal of International Business Studies 36, (pp. 304- 321)

Fox, W. (2007). Managing Organisational Behaviour. Chicago: Juta and Company Ltd. (pp.14-15).

Griffin, R., Philips, J. and Gully, S. (2012). Management cultural diversity at work Cengage Learning, (pp. 492 - 524).

Gupta, S. (2008). Mine the Potential of Multicultural Teams. HR Magazine, (pp. 79-80, 82, 84).

Jackson, S. E. \& Joshi, A. (2002). Diversity in social context: A multi attribute, multilevel analysis of team diversity and sales performance. Journal of Organizational Behavior, (pp. 675-702).

Jain, H. C. and Verma A. (1996). Workforce diversity, employment equity/affirmative action programs and public policy in selected countries. International Journal of Manpower, (pp. 14-29).

Kothari, C.R. (2010).Research methodology: Methods \& techniques. New Dehi: New Age

Miner, J. (2007), Management team learning orientation and business unit performance", Journal of Applied Psychology, (Vol. 88, pp. 552 - 60).

Mugenda, O. M. \& Mugenda, A. G. (2003) Research Methods: Quantitative and Qualitative Approaches. Nairobi: African Centre for Technology Studies (ACTS) Press.

Munjuri, M. and Maina, R. (2013), Workforce Diversity Management and Employee Performance in the Banking Sector in Kenya, (UoN Portal > Vol 3, No 1)

Webber, S.S.; Donahue, L.M. (2001) Impact of Highly and Less Job-related Diversity on Work Group Cohesion and Performance: A Meta-analysis, Journal of Management, (pp. 141-162).

Zgourides, G. D., Johnson, L., \& Watson, W. E. (2002). The influence of ethnic diversity on leadership, group process, and performance: an examination of learning teams, (pp.141-162). 